\title{
Interfaces adaptativas tangíveis e virtuais para ensino de lógica
}

\author{
Daniel Almeida Chagas \\ University of Fortaleza \\ (UNIFOR) \\ Fortaleza-CE, Brazil \\ prof.daniel.chagas@gmail.com
}

\author{
Elizabeth Sucupira Furtado \\ University of Fortaleza \\ (UNIFOR) \\ Fortaleza-CE, Brazil \\ elizabethsfur@gmail.com
}

\author{
Rafaela Ponte Lisboa \\ University of Fortaleza \\ (UNIFOR) \\ Fortaleza-CE, Brazil \\ rafaelapontelisboa@gmail.com
}

\begin{abstract}
A presente pesquisa busca trazer uma releitura dos trabalhos de Papert na criação de uma linguagem de programação para crianças, à luz da educação tecnológica (STEM) e das interfaces tangíveis. Propõe a criação, avaliação e adoção de artefatos físicos e virtuais no ensino de lógica e matemática para crianças, adaptadas a diferentes perfis de idade, aderentes aos requisitos de ensino da realidade escolar brasileira. Busca também investigar práticas para apoio ao professor na adoção de tecnologias na sala de aula.
\end{abstract}

\section{Author Keywords}

User experience; educational objects; teaching logic, stem.

\section{ACM Classification Keywords}

H.5.m. Information interfaces and presentation (e.g., $\mathrm{HCI}$ ): Miscellaneous.

\section{CARACTERIZAÇÃO DO PROBLEMA}

Acompanhamos toda uma tendência mundial no ensino, baseada na adoção de tecnologias na sala de aula. É o chamado ensino STEM - Ciência (Science), Tecnologia, Engenharia e Matemática. Essa tendência visa tratar a educação de forma mais prática e aplicada, desde o ensino fundamental até o superior. Vários estudos [6], declaram a importância do ensino tecnológico para fomento da inovação e pesquisa. Dentre elas, estão: o ensino de programação para idades cada vez menores, a aplicação de robótica e automação para ensino, e a criação de ferramentas específicas para auxiliar no ensino.

A adoção de incentivos ao ensino por meio da tecnologia para criança aumenta o interesse também do público feminino [5], onde temos que meninas decrescem seu interesse nas áreas tecnológicas e exatas. Uma dificuldade

Permission to make digital or hard copies of all or part of this work for personal or classroom use is granted without fee provided that copies are not made or distributed for profit or commercial advantage and that copies bear this notice and the full citation on the first page. Copyrights for components of this work owned by others than the author(s) must be honored. Abstracting with credit is permitted. To copy otherwise, or republish, to post on servers or to redistribute to lists, requires prior specific permission and/or a fee. Copyright 2018 SBC.

IHC 2018, Anais Estendidos do XVII Simpósio Brasileiro sobre Fatores Humanos em Sistemas Computacionais Outubro 22-26, 2018, Belém, Brasil WTD-IHC inerente ao ato de ensinar programação é o caráter abstrato da lógica [4]. Uma solução é a adoção de práticas para 'materializar' os conceitos de programação [13], como uso da própria tecnologia[10], programação em blocos e robótica. Tais abordagens têm mostrado dados promissores, principalmente quando adotados em idades menores [14] e para público feminino [5].

Pesquisas mostram as vantagens de utilização de interação tangível na educação, como em Posada e Baranauskas [18] que um ambiente sócio-construcionista, de baixo custo, para a criação de estórias pelas crianças.

Estudos da União Europeia [3] declaram a necessidade de se definir uma idade ótima de introdução dos conceitos de pensamento computacional, contudo expõem o consenso que estas capacidades devem ser introduzidas mesmo para crianças muito pequenas.

Soluções comerciais para ensino de robótica, como as da Lego são caras mesmo para escolas particulares, limitando seu uso para grupos específicos de alunos. Para as escolas públicas, tal produto tem o custo proibitivo, dado que se reflete na Olimpíada Brasileira de Robótica: Na falta de uma plataforma melhor para as competições, muitas das competições exigem que a escola possua o kit LEGO, inviabilizando a ida da grande maioria das escolas [16].

A presente pesquisa busca o desenvolvimento de objetos educacionais interativos e adaptativos para o ensino de lógica de programação e de matemática, por meio de interfaces tangíveis, que aportam um caráter prático, material, lúdico e fácil de usar. O público alvo são crianças iletradas até adolescentes, de custo acessível tanto para escolas públicas quanto particulares.

Contribuições para a pesquisa em Interação Humano Computador são descritas neste texto.

\section{FUNDAMENTAÇÃO TEÓRICA}

Os trabalhos de Papert [17] na década de 80 são os fundamentos do ensino STEM. O autor é o criador da linguagem de programação infantil Logo, que utiliza comandos simples e conhecidos (para frente, direita, esquerda, etc.) das crianças para movimentar uma tartaruga robô (física ou virtual). A linguagem Logo é utilizada como base para uma gama de softwares e plataformas modernos de ensino de lógica de programação [15]. 
Interfaces de usuário tangíveis pertencem à área de estudo da interação humano computador que utiliza artefatos tangíveis (físicos) para representar informação [19]. Aproveita-se a capacidade humana de múltiplos sentidos para manipular objetos físicos que representam informação virtual, naturalizando a interação com dispositivos tecnológicos.

\section{PROPOSTA}

Desenvolver um sistema multi-plataforma via web, aplicativo móvel e por interação tátil, que viabilize o ensino de programação, sendo a primeira plataforma em formato de cursos para formação pedagógica e a segunda, chamada Code Dominó, baseada em artefatos físicos e pictóricos e no uso de um aplicativo móvel.

\section{OBJETIVOS ESPECÍFICOS}

- Incrementar artefatos físicos, no formato de peças de dominó, com codificação embutida que seja lido por dispositivo computacional;

- Desenvolver uma linguagem de programação tangível baseada na linguagem Logo [17];

- Desenvolver um mini robô, que leia os artefatos físicos e execute os comandos programados;

- Desenvolver o aplicativo móvel que permita a manipulação de peças virtuais de programação;

- Executar testes de usabilidade e de acessibilidade com crianças de diferentes faixas etárias entre os diferentes artefatos criados; e

- Desenvolver um sistema interativo de formação pedagógica para professores e alunos.

\section{METODOLOGIA}

A metodologia do estudo compõe-se dos seguintes passos:

1. Realização de uma revisão sistemática sobre objetos educacionais tecnológicos, ou de ensino de lógica de programação, buscando analisar as principais abordagens acadêmicas e comerciais;

2. Definição de requisitos para o ensino de lógica e matemática através de objetos educacionais tecnológicos. Definir também uma subcategoria de requisitos para a realidade educacional brasileira;

3. Construção de artefatos baseados nos requisitos levantados, virtuais e tangíveis;

4. Realização de testes de usabilidade com usuários (crianças, pré-adolescentes e adolescentes), buscando avaliar os artefatos (objetos educacionais) na sua eficiência, eficácia e satisfação em diferentes faixas de idade. Questões éticas estão sendo tratadas com o apoio da Coética, setor da universidade onde esta pesquisa está sendo conduzida.

5. Realização de entrevistas e grupos de trabalho com professores, buscando relatar práticas aderentes às atuais metodologias pedagógicas que insiram os artefatos na realidade do professor.
Os primeiros dois passos já foram realizados. O projeto está na fase de refinamento e pré-testes dos artefatos interativos.

\section{REVISÃO SISTEMÁTICA}

A revisão sistemática seguiu o modelo de Nakagawa [7] para a obtenção e análise dos artigos publicados no período entre 2000 e 2017, prioritariamente no idioma inglês. O objetivo foi adquirir conhecimentos sobre outros projetos acadêmicos e científicos que visassem o ensino de lógica de programação, principalmente para alunos do ensino fundamental e médio, com diferentes objetos educacionais, nos idiomas Português e Inglês.

Sem entrar em detalhes, o resultado do processo de RS foram 20 artigos, que trazem diferentes abordagens para o ensino tecnológico. Eles podem ser agrupados em:

\section{Interação por manipulação}

A substituição dos comandos digitados por blocos de comando tem sido uma estratégia para ensino de programação tanto para crianças quanto adultos. Ao arrastar e conectar peças representando comandos e parâmetros, várias características ideais de interação são favorecidas [2, 18], como visibilidade (organização das peças, uso de tabuleiros), reconhecimento ao invés de memorização (pelas características de design), e restrições (as formas dos blocos ou peças já deixam claro que opções de combinação são viáveis ou não). Testes com 100 alunos usando programação em bloco [11], mostraram que a maioria deles conseguia prever, entender e aplicar o comportamento de um bloco, mesmo sem nunca tê-lo visto.

\section{Feedback lúdico e uso de Robôs}

$\mathrm{O}$ ensino de programação de adultos inicia-se com comandos para saídas lógico-matemáticas. $\mathrm{O}$ aluno é capacitado a operar comandos e receber respostas do computador. As práticas com crianças tratam as respostas de forma lúdica, algo mais visível ou tangível. Os movimentos físicos ou virtuais, robóticos (de cabeça, de corpo, de braços) $[2,18]$, tornam os feedback mais atrativos para crianças e adolescentes.

\section{Comandos Simples e Pictográficos}

$\mathrm{O}$ ato de programar um computador é uma interação do tipo instrução [2], digitando comandos previamente definidos, em um conjunto de regras de posicionamento do código, que não são similares nem à escrita nem à matemática. Ela assim é definida por ser um ambiente que exige a interação eficiente por parte dos programadores avançados. A dificuldade do aluno iniciante em não saber o que pode digitar, nem como organizar esses comandos é o que faz com que uma tela de um ambiente para desenvolvimento seja uma primeira frustração [12].

Ao substituir os comandos digitados por blocos, físicos ou virtuais, pictográficos, e generalistas em suas funções, identifica-se o princípio de design affordance [2] definido como "um atributo de um objeto que permite que as pessoas saibam como utilizá-lo". A manipulação de blocos de comando, com conectores, traz um affordance similar ao de 
jogos de cartas, quebra-cabeças ou dominós, quebrando a barreira da interação inicial. Evitam-se com isso erros de digitação e sintaxe, tão comuns aos iniciantes.

\section{Custo e Propriedade}

Soluções tangíveis naturalizam a interação, porém são mais caras de se adquirir que soluções baseadas somente em software. A adoção de tecnologias livres de fácil replicação pelos próprios professores diminuem esse custo, consequentemente facilitam a implementação.

\section{REQUISITOS}

A elaboração dos requisitos parte da análise das referências estudadas no estado da arte, da crítica pedagógica dessas referências, e do estudo empírico dos autores, com experiência no ensino tecnológico de adolescentes e universitários. São eles:

- Apresentar os comandos de programação não como texto, mas como blocos de comando, reais ou virtuais;

- Iniciar com códigos que gerem ludicidade (movimento, som, luz, etc.), utilizando dispositivos físicos interativos básicos, enquanto que viabiliza o progresso do aluno, utilizando recursos mais avançados (Comandos condicionais, funções, etc.);

- Apoiar o professor com planos de ensino com uma metodologia que permita explorar os artefatos (Recursos tangíveis e em diversas plataformas) de forma adaptativa ao plano de ensino.

\section{Realidade Brasileira}

Para além do conjunto de requisitos básicos apresentados, a realidade brasileira das escolas precisa de um subconjunto específico de requisitos. Isso se dá pela pouca estrutura física e de laboratórios das escolas brasileiras em geral, e pela falta de formação dos professores para adoção de tecnologias na sala de aula. São eles:

- Baixo custo de implantação, com softwares e hardwares livres (open source) que permitam a interação virtual com os elementos da proposta, ou uso de materiais de baixo custo para objetos tangíveis, como madeira M.D.F., E.V.A. e papel, e.

\section{SOLUÇÕES INTERATIVAS}
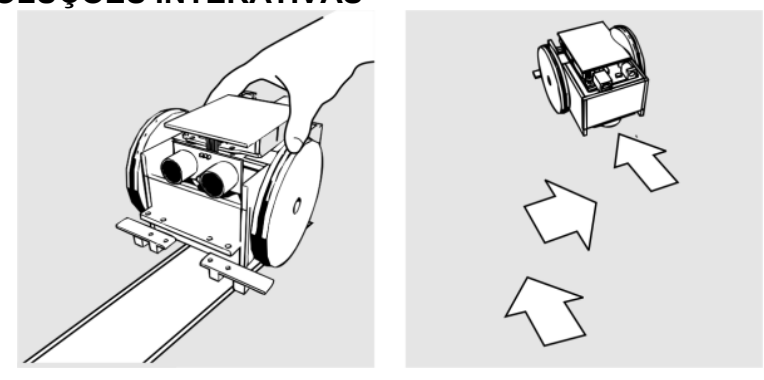

Figura 1 - Programação do robô através de peças tangíveis

\section{Soluções Tangíveis}

Foi desenvolvido um robô de baixo custo, capaz de ler uma sequência de peças RFID dispostas pelas crianças, representado os comandos (Figura 1). Em seguida o robô executa as instruções lidas. Esta modalidade de interação tangível (sem uso de telas ou representações virtuais) favorece a colaboração entre as crianças.

Dois conjuntos de peças de dominó foram desenvolvidos (Figura 2), para diferentes faixas de idade: $\mathrm{O}$ primeiro traz somente instruções concretas de movimentação do robô (frente, trás, direita, esquerda, etc.), enquanto o segundo traz elementos presentes na lógica de programação (operações, parâmetros, condicionais, funções, etc.).
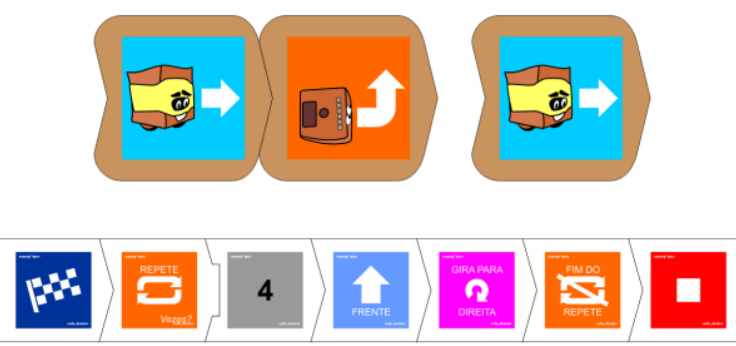

Figura 2 - Diferentes peças de comandos do robô.

\section{Soluções Virtuais}

Um aplicativo para tablets está em desenvolvimento buscando trazer novas formas de interação o projeto. Também são apresentadas duas formas de interação virtual, para diferentes faixas de idade: Modo desenho, onde a criança desenha o caminho que o robô deve percorrer, que é depois transformado em peças virtuais e enviado ao robô para execução; e modo dominó virtual, onde ela manipula peças de comando virtuais, podendo criar sequências mais longas que com o dominó tangível.

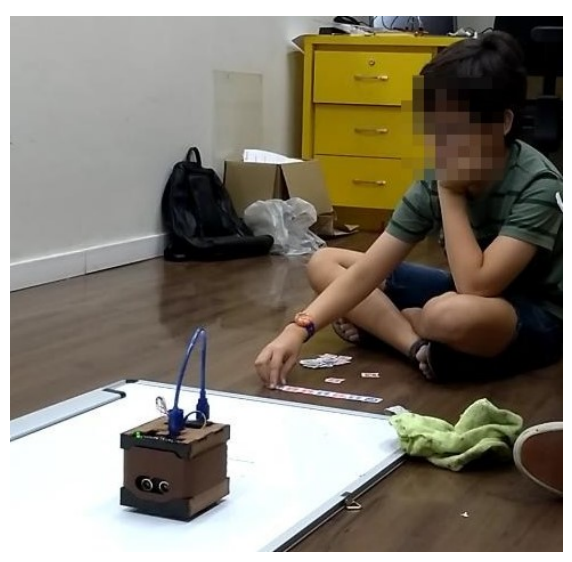

Figura 3. Uso do dominó e robô sobre um quadro de lousa para facilitar o movimtento

\section{RESULTADOS PRELIMINARES}

Dois conjuntos de peças de dominó (para iletrados e crianças) foram desenvolvidos. São formados por peças em acrílico, contendo uma etiqueta RFID e adesivo impresso com a função da peça. O conjunto de peças para os iletrados contem somente os comandos básicos de movimento do robô. O dominó para crianças, além dos comandos básicos de movimento, inclui os comandos vistos em linguagens de 
programação modernas, como estruturas de repetição, estruturas condicionais, variáveis e funções.

Testes pilotos de usabilidade foram realizados com o robô e o dominó de programação com 3 crianças (Figura 33 ).

Este trabalho visa contribuir para a pesquisa em Interação Humano Computador à medida que usa os desafios lançados pela comunidade no documento GranDIHC [1], destacando:

- Acessibilidade e Inclusão Digital, com o desenvolvimento e inovação com a temática de Tecnologias Assistivas, Adaptáveis e Inclusivas [8]. A substituição dos comandos digitados por blocos de comando contendo objetos interativos educacionais desenvolvidos e a substituição de plataformas caras e importadas por soluções de custo menor tem os objetivos de ampliar a capacidade de auxiliar no ensino o ensino das áreas STEM, e contribuir para a igualdade social e de gênero no trabalho;

- Ubiquidade, Múltiplos Dispositivos e Tangibilidade [9], com, artefatos físicos de interação mais acessíveis, e interfaces tangíveis mais contextualizadas as diversas realidades de interação;

\section{REFERÊNCIAS BIBLIOGRÁFICAS}

1. Baranauskas, M.C.C. et al. 2012. GranDIHC-BR: Prospecção De Grandes Desafios De Pesquisa Em Interação Humano-computador No Brasil. Companion Proceedings of the 11th Brazilian Symposium on Human Factors in Computing Systems (Porto Alegre, Brazil, Brazil, 2012), 63-64.

2. Barbosa, S. and Silva, B. 2010. Métodos de Avaliaçao de IHC. Interaçao Humano-Computador. Elsevier. 384.

3. Bocconi, S. et al. 2016. Developing computational thinking in compulsory education. Proceedings EdMedia 2016.

(2016).

DOI:https://doi.org/10.2791/792158.

4. Bosse, Y. and Gerosa, M.A. 2017. Why is Programming So Difficult to Learn?: Patterns of Difficulties Related to Programming Learning Mid-Stage. SIGSOFT Softw. Eng. $\quad$ Notes. 41, 6 (2017), 1-6. DOI:https://doi.org/10.1145/3011286.3011301.

5. Commission On Technology, Gender, A.T.E. 2000. Tech-Savvy: Educating Girls in the New Computer Age.

6. Dopplick, R. 2015. Maker Movement and Innovation Labs. ACM Inroads. 6, 4 (Nov. 2015), 108. DOI:https://doi.org/10.1145/2829975.

7. Elisa Nakagawa 2017. Revisão Sistemática da Literatura em Engenharia de Software.

8. Furtado, E.. et al. 2012. Acessibilidade e Inclusão Digital. I GranDIHC-BR - Grandes Desafios de Pesquisa em Interação Humano-Computador no Brasil. C.E. de I.H.-C. CEIHC, ed. Sociedade Brasileira de Computação. 19-22.

9. Furtado, E.. et al. 2012. Ubiquidade, Múltiplos
Dispositivos e Tangibilidade. I GranDIHC-BR Grandes Desafios de Pesquisa em Interação HumanoComputador no Brasil. C.E. de I.H.-C. CEIHC, ed. Sociedade Brasileira de Computação. 23-26.

10. Guimarães, R.L. et al. 2017. A Comparative Study of Technology-Mediated Learning Strategies for Teaching People with Intellectual Disability. Proceedings of the XVI Brazilian Symposium on Human Factors in Computing Systems (New York, NY, USA, 2017), 72:1$-72: 4$.

11.Honig, W.L. 2013. Teaching and assessing programming fundamentals for non majors with visual programming. Proceedings of the 18th ACM conference on Innovation and technology in computer science education - ITiCSE '13 (New York, NY, USA, 2013), 40.

12. Horn, M.S. et al. 2009. Comparing the use of tangible and graphical programming languages for informal science education. Proceedings of the 27th international conference on Human factors in computing systems CHI 09. (2009), 975. DOI:https://doi.org/10.1145/1518701.1518851.

13. Kalelioğlu, F. 2015. A new way of teaching programming skills to K-12 students: Code.org. Computers in Human Behavior. 52, (2015), 200-210. DOI:https://doi.org/10.1016/j.chb.2015.05.047.

14. Katterfeldt, E.-S. et al. 2016. Talkoo. A new paradigm for physical computing at school. Proceedings of the The 15th International Conference on Interaction Design and Children - IDC '16. (2016), 512-517. DOI:https://doi.org/10.1145/2930674.2935990.

15. McNerney, T.S. 2004. From turtles to Tangible Programming Bricks: Explorations in physical language design. Personal and Ubiquitous Computing. 8, 5 (2004), 326-337. DOI:https://doi.org/10.1007/s00779004-0295-6.

16. de Miranda, L.C. et al. 2010. RoboFácil: Especificação e Implementação de um Kit de Robótica para a Realidade Educacional Brasileira. Revista Brasileira de Informática na Educação. (2010). DOI:https://doi.org/10.5753/RBIE.2010.18.03.46.

17. Papert, S. 1993. The Children's Machine: Rethinking School in the Age of the Computer. Basic Books, Inc.

18. Posada, J.E.G. and Baranauskas, M.C.C. 2015. A Socioconstructionist Environment to Create Stories Using Tangible Interfaces. Proceedings of the 14th Brazilian Symposium on Human Factors in Computing Systems (New York, NY, USA, 2015), 1:1--1:10.

19. Tangible Media Group, 2006. Tangibles at play. $A C M$ SIGGRAPH 2006 Emerging technologies on SIGGRAPH '06. 32. DOI:https://doi.org/10.1145/1179133.1179166. 